\title{
Long GRBs from binary stars: runaway, Wolf-Rayet progenitors
}

\author{
M.Cantiello*, S.-C.Yoon ${ }^{\dagger}$, N.Langer* and M.Livio** \\ *Astronomical Institute, Utrecht University, \\ Princetonplein 5, 3584 CC, Utrecht, The Netherlands \\ ${ }^{\dagger}$ Department of Astronomy and Astrophysics, University of California, Santa Cruz, CA 95064, USA \\ ${ }^{* *}$ Space Telescope Science Institute, 3700 San Martin Drive, Baltimore, MD 21218
}

\begin{abstract}
The collapsar model for long gamma-ray bursts requires a rapidly rotating Wolf-Rayet star as progenitor. We test the idea of producing rapidly rotating Wolf-Rayet stars in massive close binaries through mass accretion and consecutive quasi-chemically homogeneous evolution - the latter had previously been shown to provide collapsars below a certain metallicity threshold for single stars. The binary channel presented here may provide a means for massive stars to obtain the high rotation rates required to evolve quasi-chemically homogeneous and fulfill the collapsar scenario. Moreover, it suggests that a possibly large fraction of long gamma-ray bursts occurs in runaway stars.
\end{abstract}

Keywords: Stars: binary - Stars: rotation - Stars: evolution - Stars: mass-loss - Supernovae: general - Gamma rays: bursts

PACS: 97.

\section{INTRODUCTION}

Long gamma-ray bursts are thought to be produced by a subset of dying massive and possibly metal-poor stars [1, 2, 3]. Within the currently favored collapsar scenario [4], the burst is produced by a rapidly rotating massive Wolf-Rayet (WR) star whose core collapses into a black hole [5]. While single star evolution models without internal magnetic fields can produce such configurations [6, 7], only models including magnetic fields are capable of reproducing the slow spins of young Galactic neutron stars [8, 9] and white dwarfs [10], due to the magnetic core-envelope coupling during the giant stage.

Yoon and Langer [11], Yoon et al. [12] and Woosley and Heger [13] recently showed that below a certain metallicity threshold, very rapidly rotating single stars avoid the magnetic braking of the core through the so-called quasi-chemically homogeneous evolution: rotationally induced mixing processes keep the star close to chemical homogeneity, and thus the giant stage is avoided altogether. While these models are successful in producing models which fulfill all constraints of the collapsar model, they require very rapid initial rotation.

The question thus arises whether the quasi-chemically homogeneous evolution of massive stars can also be obtained in mass transferring massive binary systems [14], since in such systems the mass gainer can be spun-up to close to critical rotation [see 15 , 6], independent of its initial rotation rate. 

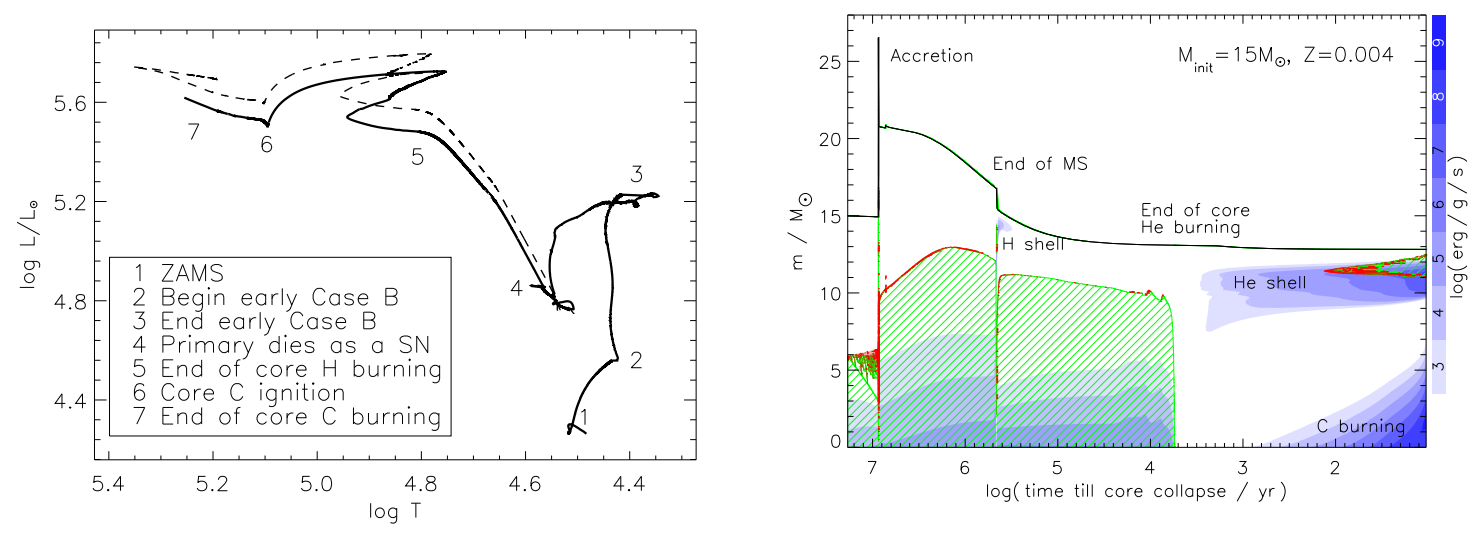

FIGURE 1. Left panel: evolutionary track of the mass gainer in our $16 \mathrm{M}_{\odot}+15 \mathrm{M}_{\odot}$ early Case B binary model ( $5 \mathrm{~d}$ initial orbital period) in the HR diagram (solid line), from the zero age main sequence up to core carbon exhaustion. The main evolutionary phases are labeled by numbers (see legend). The dashed line shows the evolutionary track of a very rapidly rotating $\left(v_{\text {init }} / v_{\mathrm{K}}=0.9\right) 24 \mathrm{M}_{\odot}$ single star. Both stars have SMC metallicity, and undergo quasi-chemically homogeneous evolution (see text). Right panel: evolution of the internal structure of the mass gainer of the computed $16 \mathrm{M}_{\odot}+15 \mathrm{M}_{\odot}$ early Case B binary sequence, as function of time, from the zero-age main sequence to core carbon exhaustion. The time axis is logarithmic, with the time of core collapse as zero point. Convective layers are hatched. Semiconvective layers are marked by dots (red dots in the electronic version). Gray (blue) shading indicates nuclear energy generation (color bar to the right of the figure). The topmost solid line denotes the surface of the star.

\section{THE MODEL}

We use a 1-D hydrodynamic binary evolution code to simulate the evolution of a $16+15$ $\mathrm{M}_{\odot}$ binary model with an initial orbital period of 5 days and SMC metallicity $(Z=0.004)$. Internal differential rotation, rotationally induced mixing and magnetic fields are included in both components, as well as non-conservative mass and angular momentum transfer, and tidal spin-orbit coupling. Detailed description of the code and of the adopted physics can be found in Cantiello et al. [16] and references therein.

We chose an early Case B system with an initial mass ratio close to one for two reasons. Firstly, the expected mass transfer efficiency for this case was about $60 \%$ (meaning that $60 \%$ of the transfered matter can be retained by the mass gainer), based on the calculations by Wellstein [17], Langer et al. [18], and Petrovic et al. [15]. Secondly, a Case B rather than Case A system was chosen to avoid synchronization after the major mass transfer phase.

\section{RESULTS}

The evolution of the binary system proceeded as follows (cf. Table 1). The initial rotational velocity of both stars has been set to $230 \mathrm{~km} \mathrm{~s}^{-1}$, but both stars synchronize with the orbital rotation within about $1 \mathrm{Myr}$, to equatorial rotational velocities of only about $50 \mathrm{~km} \mathrm{~s}^{-1}$. Rotationally induced mixing before the onset of mass transfer is thus negligible - in contrast to typical O stars evolving in isolation [19, 20]. The initially 
TABLE 1. Major evolutionary phases of the computed $16 \mathrm{M}_{\odot}+15 \mathrm{M}_{\odot}$ early Case B binary sequence. The binary calculation ends after core carbon exhaustion of the mass loser (the primary), and the mass gainer (the secondary) is then evolved as a single star. We show evolutionary time, masses of both stars, orbital period, surface rotational velocity of both stars, surface and core helium mass fraction of the mass gainer, and orbital velocity of the mass gainer. The abbreviations for the evolutionary phases are: ZAMS = zero age main sequence; $\mathrm{ECHB}=$ end core hydrogen burning; $\mathrm{ICB}=$ ignition of carbon burning; ECCB $=$ end core carbon burning. The numbered evolutionary stages correspond to those given in Fig. 1, left panel.

\begin{tabular}{l|ccccccccc}
\hline Phase & $\begin{array}{c}\text { Time } \\
\mathrm{Myr}\end{array}$ & $\begin{array}{c}\mathrm{M}_{1} \\
\mathrm{M}_{\odot}\end{array}$ & $\begin{array}{c}\mathrm{M}_{2} \\
\mathrm{M}_{\odot}\end{array}$ & $\begin{array}{c}\mathrm{P} \\
\mathrm{d}\end{array}$ & $\begin{array}{c}v_{\text {rot, }, 1} \\
\mathrm{~km} \mathrm{~s}^{-1}\end{array}$ & $\begin{array}{c}v_{\text {rot }, 2} \\
\mathrm{~km} \mathrm{~s}^{-1}\end{array}$ & $\mathrm{Y}_{\mathrm{c}, 2}$ & $\mathrm{Y}_{\mathrm{s}, 2}$ & $\begin{array}{c}v_{\text {orbit, } 2} \\
\mathrm{~km} \mathrm{~s}^{-1}\end{array}$ \\
\hline 1 ZAMS & 0 & 16 & 15 & 5.0 & 230 & 230 & 0.248 & 0.248 & 201 \\
2 begin Case B & 9.89 & 15.92 & 14.94 & 5.1 & 96 & 85 & 0.879 & 0.248 & 198 \\
3 end Case B & 9.90 & 3.93 & 20.77 & 38.2 & 27 & 719 & 0.434 & 0.348 & 29 \\
4 ECCB primary & 11.30 & 3.71 & 20.86 & 42.7 & 40 & 767 & 0.457 & 0.441 & 27 \\
& & & & & & & & & \\
5 ECHB secondary & 18.10 & - & 16.76 & - & - & 202 & 0.996 & 0.956 & - \\
6 ICB secondary & 18.56 & - & 12.85 & - & - & 191 & 0.000 & 0.996 & - \\
7 ECCB secondary & 18.56 & - & 12.83 & - & - & 258 & 0.000 & 0.996 & - \\
\hline
\end{tabular}

more massive star ends core hydrogen burning after $\sim 9.89 \mathrm{Myr}$, and Case B mass transfer begins shortly thereafter. It sheds about $12 \mathrm{M}_{\odot}$ evolving into a $\sim 4 \mathrm{M}_{\odot}$ helium star. About $1.5 \mathrm{Myr}$ later, it sheds another $\sim 0.2 \mathrm{M}_{\odot}$ as a helium giant, before exploding as Type Ib/c supernova.

The mass gainer keeps about $6 \mathrm{M}_{\odot}$ of the overflowing matter, rendering the mass accretion efficiency to roughly $50 \%$. Thereafter, it enters a phase of close-to-critical rotation, which induces rejuvenation and quasi-chemically homogeneous evolution (Fig. 1). Its mass loss is enhanced by rotation. About $5 \mathrm{Myr}$ after the onset of accretion, the surface helium mass fraction of the mass gainer is increased to values above $60 \%$, and Wolf-Rayet mass loss is assumed from then on. The star finishes core hydrogen burning after another $3 \mathrm{Myr}$, at an age of $18.1 \mathrm{Myr}$, with a mass of $16.8 \mathrm{M}_{\odot}$, a surface helium mass fraction of $95 \%$, and rotating with $\sim 200 \mathrm{~km} \mathrm{~s}^{-1}$ at the surface.

After core hydrogen exhaustion, the mass gainer contracts and spins-up to critical rotation, which leads to a mass shedding of almost $2 \mathrm{M}_{\odot}$. During its remaining lifetime of less than $0.5 \mathrm{Myr}$, it loses about another $2 \mathrm{M}_{\odot}$ to a Wolf-Rayet wind. It ends its life as a rapidly rotating Wolf-Rayet star with a final mass of about $13 \mathrm{M}_{\odot}$, ready to form a collapsar (average specific angular momentum in the $\mathrm{CO}$ core $<j_{\mathrm{CO}}>\simeq 2 \times 10^{16}$ $\mathrm{cm}^{2} \mathrm{~s}^{-1}$ ). Assuming the binary brakes up due to the explosion of the mass loser, the mass gainer would have traveled for about $7 \mathrm{Myr}$ with its final orbital velocity of $27 \mathrm{~km} \mathrm{~s}^{-1} \mathrm{a}$ distance of about $200 \mathrm{pc}$.

\section{DISCUSSION}

The binary evolution model presented above shows that quasi-chemically homogeneous evolution may occur in mass gainers of low-metallicity massive early Case B binaries. The comparison of the mass gainer with a corresponding single star model made it clear that such binary components evolve in the same way as extremely rapidly rotating single 
stars. This confirms that the scenario of quasi-chemically homogeneous evolution might not be restricted to single stars, but may apply to the accreting component of massive close binaries as well.

While we provide only one example, it seems likely that this scenario applies to most massive close binary components which accrete or gain an appreciable amount of mass; this may encompass Case A binaries and early Case B binaries [21, 22, 23]. Case A merger are also likely contributing to this scenario. While the merged object will have more mass than the initially more massive star in the binary, the product will be extremely rapidly rotating due to the orbital angular momentum, as in the case of some blue stragglers [24].

\section{Binaries and the distribution of rotational velocities}

The best constraint so far on the distribution of initial rotational velocities (IRF) comes from the recent study of young $\mathrm{O}$ stars in the SMC, mostly from the cluster NGC 346 [25]. According to Yoon et al. [12], the three most rapid rotators from the sample of $21 \mathrm{O}$ stars would qualify for the quasi-chemically homogeneous evolution scenario, and remarkably, all three stars are found to be helium-enhanced. The simplest approach to understand those stars is to assume that they correspond to the tip of the IRF.

However, that data of Mokiem et al. [25] reveals another interesting feature: two of the the three mentioned stars are runaway stars, with radial velocities deviating by $30 \ldots 70 \mathrm{~km} \mathrm{~s}^{-1}$ from the average cluster radial velocity. While dealing with low number statistics, this information opens another possibility: that the most rapidly rotating young $\mathrm{O}$ stars in the SMC are products of binary evolution. A closer examination of the IRF derived by Mokiem et al. [25] appears to support this idea: While the three rapid rotators show $v \sin i \gtrsim 290 \mathrm{~km} \mathrm{~s}^{-1}$, all other stars have $v \sin i \lesssim 210 \mathrm{~km} \mathrm{~s}^{-1}$.

The following hypothesis therefore seems conceivable: The IRF of single $\mathrm{O}$ stars in the SMC ends at about $210 \mathrm{~km} \mathrm{~s}^{-1}$ — too early to allow quasi-chemically homogeneous evolution and collapsar formation. However, massive close binary evolution enhances the IRF to what we may call the apparent IRF as measured by Mokiem et al. [25], which leads to the redshift dependent GRB rate as worked out by Yoon et al. [12]. According to the binary population synthesis model of Podsiadlowski et al. [21], about $10 \%$ of all massive binaries might lead to a Case A merger or early Case B mass transfer, which is sufficient to populate the rapidly rotating part of the IRF of Mokiem et al. In that context, the rapidly rotating $\mathrm{O}$ star in the sample of Mokiem et al. [25] which does not appear as runaway star could either have an undetected high proper motion, or it could be the result of a Case A merger - where no runaway is produced.

\section{Effects from runaway GRBs}

The runaway nature of a GRB progenitor, as obtained in our example, has important observational consequences for both the positions of GRBs, and their afterglow prop-

erties. Concerning the afterglow, it is relevant that the medium close to a WR star has 
the density profile of a free-streaming wind, and analytical and numerical calculations both suggest that the free wind of a single WR star typically extends over many parsec [26]. However, from the analysis of GRB afterglows, a constant circumstellar medium density has been inferred in many cases [27, 28, 29, 30]. A possible explanation has been proposed by van Marle et al. [26], who simulated the circumstellar medium around a moving WR star. As the GRB jet axis is likely perpendicular to the space velocity vector, the jet escapes through a region of the bow-shock where the wind termination shock is very close to the star. Therefore, the jet may enter a constant density medium quickly in this situation.

Concerning the GRB positions, since the spin axis of the stars in a close binary system are likely orthogonal to the orbital plane, the observation of a GRB produced by the proposed binary channel is possible only if the binary orbit is seen nearly face on. Then the direction of motion of the runaway GRB progenitor must be orthogonal to the line of sight, allowing the progenitor, for the given space velocity, to obtain the maximum possible apparent separation from its formation region. The finding of Hammer et al. [31], that the nearest three long gamma-ray bursts may be due to runaway stars is in remarkable agreement with our scenario. While the collapsar progenitor in our binary model travels only $200 \mathrm{pc}$ before it dies, compared to the $400 \ldots 800$ pc deduced by Hammer et al. [31], binary evolution resulting in higher runaway velocities are certainly possible [15]. It remains to be analyzed whether the runaway scenario is compatible with the finding that long GRBs are more concentrated in the brightest regions of their host galaxies than core collapse supernovae [32].

\section{REFERENCES}

1. P. Jakobsson, G. Björnsson, J. P. U. Fynbo, G. Jóhannesson, J. Hjorth, B. Thomsen, P. Møller, D. Watson, B. L. Jensen, G. Östlin, J. Gorosabel, and E. H. Gudmundsson, MNRAS 362, 245-251 (2005).

2. $\quad$ N. Langer, and C. A. Norman, ApJL 638, L63-L66 (2006).

3. M. Modjaz, R. Kewley, R. Kirshner, K. Stanek, P. Challis, P. Garnavich, and J. Greene, J.E.and Prieto, astro-ph/0701246, AJ submitted (2007).

4. S. E. Woosley, ApJ 405, 273-277 (1993).

5. A. I. MacFadyen, and S. E. Woosley, ApJ 524, 262-289 (1999).

6. J. Petrovic, N. Langer, S.-C. Yoon, and A. Heger, AEFA 435, 247-259 (2005).

7. R. Hirschi, G. Meynet, and A. Maeder, AEFA 443, 581-591 (2005).

8. A. Heger, S. E. Woosley, and H. C. Spruit, ApJ 626, 350-363 (2005).

9. C. D. Ott, A. Burrows, T. A. Thompson, E. Livne, and R. Walder, ApJS 164, 130-155 (2006).

10. M. Suijs, N. Langer, S. C. Yoon, A. J. Poelarends, and A. Heger, in prep (2007).

11. S.-C. Yoon, and N. Langer, AEA 443, 643-648 (2005).

12. S.-C. Yoon, N. Langer, and C. Norman, $A \mathcal{F} A$ 460, 199-208 (2006).

13. S. E. Woosley, and A. Heger, ApJ 637, 914-921 (2006).

14. D. Vanbeveren, and C. de Loore, $A \mathcal{F} A$ 290, 129-132 (1994).

15. J. Petrovic, N. Langer, and K. A. van der Hucht, $A \mathcal{E} A$ A 435, 1013-1030 (2005).

16. M. Cantiello, S.-C. Yoon, N. Langer, and M. Livio, AEFA 465, L29-L33 (2007).

17. S. Wellstein, PhD thesis, University of Potsdam (2001).

18. N. Langer, S.-C. Yoon, J. Petrovic, and A. Heger, "Binary evolution models with rotation," in $I A U$ Symposium, edited by A. Maeder, and P. Eenens, 2004, p. P. 535.

19. A. Heger, and N. Langer, ApJ 544, 1016-1035 (2000).

20. G. Meynet, and A. Maeder, AEFA 361, 101-120 (2000).

21. P. Podsiadlowski, P. C. Joss, and J. J. L. Hsu, ApJ 391, 246-264 (1992). 
22. S. Wellstein, and N. Langer, AEFA 350, 148-162 (1999).

23. S. Wellstein, N. Langer, and H. Braun, AEA 369, 939-959 (2001).

24. M. Livio, "Blue Stragglers: The Failure of Occam's Razor?," in ASP Conf. Ser. 53: Blue Stragglers, edited by R. A. Saffer, 1993, p. 3.

25. M. R. Mokiem, A. de Koter, C. J. Evans, J. Puls, S. J. Smartt, P. A. Crowther, A. Herrero, N. Langer,

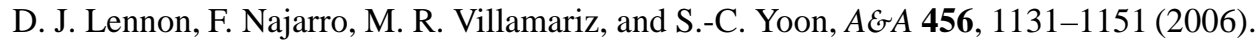

26. A. J. van Marle, N. Langer, A. Achterberg, and G. Garcaía-Segura, AEA 460, 105-116 (2006).

27. R. A. Chevalier, and Z.-Y. Li, ApJ 536, 195-212 (2000).

28. A. Panaitescu, and P. Kumar, ApJ 554, 667-677 (2001).

29. A. Panaitescu, and P. Kumar, ApJ 571, 779-789 (2002).

30. R. A. Chevalier, Z.-Y. Li, and C. Fransson, ApJ 606, 369-380 (2004).

31. F. Hammer, H. Flores, D. Schaerer, M. Dessauges-Zavadsky, E. Le Floc'h, and M. Puech, $A \mathcal{E} A$ 454, 103-111(2006).

32. A. S. Fruchter, A. J. Levan, L. Strolger, P. M. Vreeswijk, S. E. Thorsett, D. Bersier, I. Burud, J. M. Castro Cerón, A. J. Castro-Tirado, C. Conselice, T. Dahlen, H. C. Ferguson, J. P. U. Fynbo, P. M. Garnavich, R. A. Gibbons, J. Gorosabel, T. R. Gull, J. Hjorth, S. T. Holland, C. Kouveliotou, Z. Levay, M. Livio, M. R. Metzger, P. E. Nugent, L. Petro, E. Pian, J. E. Rhoads, A. G. Riess, K. C. Sahu, A. Smette, N. R. Tanvir, R. A. M. J. Wijers, and S. E. Woosley, Nature 441, 463-468 (2006). 